The Egyptian Journal of Hospital Medicine (April 2019) Vol. 75 (6), Page 3139-3142

\title{
The Role of Acetic Acid Chromoendoscopy and Narrow Band Imaging in Diagnosis of Esophageal Barrett Dysplasia
}

\author{
Magdy Eldahshan ${ }^{1}$, Ashraf Albahrawy ${ }^{1}$, Zakarya Mohamed Zkareya ${ }^{1}$, Shadia Hussein Mabrouk ${ }^{2}$, Alaa Alsawak ${ }^{1}$ \\ 1 Department of Internal Medicine, Faculty of Medicine, Al-Azhar University, \\ 2 Department of Pathology, Faculty of Medicine, Ain Shams University, Cairo, Egypt \\ Corresponding author: Alaa Alsawak, email: alaaalsawak5@gmail.com
}

\section{ABSTRACT}

Background: Barrett's esophagus (BE) is an important risk factor for the occurrence esophageal adenocarcinoma. Endoscopic surveillance for Barrett's dysplasia is indicated in all patients with BE. Recent data found that with the use of advanced endoscopic techniques there is an increased diagnostic yield for dysplasia.

Aim: The aim of this study is to assess the accuracy of acetic acid assisted narrow band imaging (AA-NBI) endoscopy in detection of Barrett's dysplasia. Patients and Methods: Forty patients with BE surveyed for Barrett's dysplasia by AANBI endoscopy. Patients with difficult sampling $(n=1)$ or not proved to have Barrett's mucosa by histopathology $(n=5)$ were excluded. Patients with positive and negative dysplasia underwent targeted and random endoscopic biopsy, respectively. The diagnosis of BE based on Prague endoscopic classification. The pathological assessment of Barrett's dysplasia based on Vienna classification. Results: A total of 34 patients were included; their mean age was $39.03 \pm 14.23$ years; they were $19(55.88 \%)$ males and $15(44.11 \%)$ females. Of them $28(82.35 \%)$ had cardiac type BE, $3(8.82 \%)$ had fundic type BE and 3 (8.82\%) had intestinal type BE. Five patients were depicted as Barrett's dysplasia by AA-NBI. Low grade dysplasia was proved in 3 of them by histopathology. The sensitivity, specificity, positive predictive value, negative predictive value and accuracy of AA-NBI in diagnosis of Barrett's dysplasia were 100\%, 93.5\%, 60\%, 100\%, and 94.1\% respectively. Conclusion: Nine percent of patients with BE are positive for dysplasia. AA-NBI has promising sensitivity, specificity and accuracy in prediction of Barrett's dysplasia.

Keywords: Barrett's esophagus. Dysplasia. Acetic acid chromoendoscopy. Narrow band imaging. esophageal adenocarcinoma.

\section{INTRODUCTION}

The incidence of esophageal cancer is rising ${ }^{1}$ representing the seventh most common cancer worldwide. It is well known that Barrett's esophagus (BE) is considered an important risk factor for the occurrence of esophageal adenocarcinoma (EAC) and is present in up to $1.6 \%$ of the general population ${ }^{2}$ and in up to $20 \%$ of patients suffering from gastroesophageal reflux disease (GERD) ${ }^{3}$.

It is postulated that the development of EAC in Barrett occurs in a progressive fashion from intestinal metaplasia (IM) to low grade dysplasia (LGD) to high grade dysplasia (HGD) and then EAC. The annual rate of transformation into EAC in BE patients without dysplasia is estimated to be between $0.07 \%$ and $0.82 \%{ }^{4}$. While the annual rate of progression from LGD to HGD or EAC is as high as $8.8 \%^{5}$ and from HGD to EAC is $12 \%$ to $40 \%{ }^{6}$.

The aim of endoscopic surveillance for Barrett dysplasia is to change the natural history of the disease through recognizing dysplasia at an earlier stage and thus introducing beneficial endoscopic treatment. Established surveillance protocols suggest Seattle protocol with taking targeted biopsies of visible lesions and random four quadrant biopsies (4QBS) every $1-2 \mathrm{~cm}$ which seemingly offers the maximum yield of dysplasia in comparison with other biopsy protocols ${ }^{7}$. However, there are several disadvantages to this Seattle protocol including only $13 \%$ of early neoplastic lesions appearing as visible nodules ${ }^{8}$, a major proportion of Barrett's neoplasia is not visible on high definition white-light endoscopy alone, with reported sensitivity in the range $40 \%-64 \%$ and specificity $98 \%$ $100 \%$. In addition, 4QBS are time-consuming, costly and poorly accepted by patients. These drawbacks have encouraged evaluation of more effective techniques to improve the diagnostic accuracy for the detection of IM and early Barrett's dysplasia used in surveillance ${ }^{\mathbf{1 0}}$.

\section{PATIENTS AND METHODS}

The study was performed on 40 consecutive adults with $\mathrm{BE}$ who required endoscopic examination at ElHussein University Hospital between December 2017 and April 2019, Department of Internal Medicine, Gastroenterology and Hepatology Unit.

Patients with advanced esophageal cancer, previous esophageal resection, on-going treatment with antiplatelet medication, or anticoagulant medication, and those with hemorrhagic diseases, were excluded.

\section{Ethics and Patient Consent}

The study was approved by the Ethics Board of AlAzhar University and an informed written consent was taken from each participant in the study.

\section{Endoscopic procedure:}

Surveillance of BE was conducted by white light imaging (WLI), and narrow band imaging (NBI). Endoscopic diagnosis of BE was based on the detection of 
the characteristic "tongues" or salmon-pink colored extensions of mucosa that grow into the esophagus above the gastroesophageal junction using Prague criteria to determine circular and maximum length of Barrett segment ${ }^{7}$. Assessment of Barrett's dysplasia was carried on by magnified WLI, magnified NBI and acetic acid chromoendoscopy. The magnified images were analyzed with respect to pit-patterns, regular mucosal patterns (circular, ridge/villous, tubular), irregular mucosal patterns (absent or irregular) and vascular patterns (regular or irregular) using BING criteria ${ }^{\mathbf{1 1}}$. Following this, ${ }^{10}$ $20 \mathrm{~mL}$ of $2 \%$ acetic acid (AA) was sprayed by using spray catheter via the instrument channel over the lower esophagus and Barrett's epithelium. At first Barrett's mucosa took on a white appearance (the aceto-white reaction), while dysplastic mucosa was turned red earlier than the non dysplastic Barrett's mucosa ${ }^{\mathbf{1 2}}$.

The positions of the lesions detected by WLE, NBI or AA were recorded and photographed to confirm the accuracy of the biopsies obtained. At least one targeted biopsy was collected separately from the endoscopic lesions suspicious of Barrett's dysplasia by AA-NBI or WLE. In absence of Barrett's dysplasia 2-4 random biopsies were taken from the different sites of barrette's mucosa. All real time endoscopic assessment was carried by one author.

\section{Assessment of video records and photographs:}

A second session of assessment was conducted by two authors using the video records and photographs to confirm the results of real time assessment session. In case of conflict between both sessions, agreement was reached by consensus.

\section{Histopathology:}

All biopsy specimens were fixed in $4 \%$ formalin and embedded in paraffin and the slides were processed by using hematoxylin and eosin (H\&E) stains. All histologic analyses were performed by single experienced pathologist who was uninformed about the results of endoscopy. The biopsy specimens were analyzed for the presence of specialized intestinal metaplasia, dysplasia and/or cancer by using Vienna classification ${ }^{\mathbf{1 3}}$.

\section{Statistical Analysis}

For the per-patient analysis, the lesions suspected by endoscope in one patient were considered one unit of analysis in this evaluation. The sensitivity, specificity, positive predictive values, negative predictive values and accuracy for the prediction of Barrett's dysplasia in the AA-NBI were calculated using histology as a reference value.

\section{RESULTS}

This study included 40 patients diagnosed as having Barrett's Esophagus by endoscopy 5 of them were false positive endoscopic results for $\mathrm{BE}$ (negative by histopathology), the causes of this false results were chronic active reflux esophagitis $(n=3)$, chronic active reflux esophagitis with ulceration $(\mathrm{n}=1)$ and wrong sampling $(n=1)$ and 1 case was difficult to be biopsied. These 6 cases were excluded from the final analysis. The remaining 34 patients were diagnosed to have $\mathrm{BE}$ by endoscopy, which was confirmed by histopathology.

\section{Demography and clinical data:}

Among the demographic and clinical data of 34 patients with $\mathrm{BE}$ the mean age was $39.03 \pm 14.23$ years; they were $19(55.88 \%)$ males and 15 (44.12\%) females. Of them 15 patients $(44.1 \%)$ had no history of GERD while 19 patients (55.9\%) had history of GERD. 10 patients $(52.6 \%)$ had GERD < 1 year ago, 6 patients (31.6\%) had GERD $1-3$ years ago and 3 patients $(15.8 \%)$ had GERD > 3 years ago. As regard indications of endoscopy, abdominal (epigastric) pain was recorded in 19 patients, vomiting was recorded in 9 patients, bleeding per rectum was recorded in 1 patient, chronic diffuse abdominal pain was recorded in 1 patient, heart burn was recorded in 6 patients, screening for malignancy was recorded in 2 patients, melena was recorded in 1 patient, hematemesis was recorded in 1 patient, iron deficiency anemia was recorded in 2 patients, regurgitation was recorded in 2 patients and esophageal dilation was recorded in 1 patient. Smoking were recorded in 11 patients $(32.4 \%)$ while 23 patients $(67.6 \%)$ were nonsmoker.

\section{Endoscopic data:}

As regarding the endoscopic data of all patients with BE (34 patients); 30 (88.2\%) had regular mucosal pattern, $4(11.8 \%)$ had irregular mucosal pattern, $29(85.3 \%)$ had regular vascular pattern and $5(14.7 \%)$ had irregular vascular pattern on NBI by using Barrett's International NBI Group (BING) criteria, on AA-WLE and AA-NBI 29 $(85.3 \%)$ had BE with no dysplasia and $5(14.7 \%)$ had Barrett's dysplasia with no difference between AA-WLI and AA-NBI. Also GERD was present in $15(44.1 \%)$ and $19(55.9 \%)$ had no GERD on endoscopy. Among 15 patients with GERD $8(53.3 \%)$ had GERD grade B, 4 (26.7\%) had GERD grade A and 3 (20\%) had GERD grade $\mathrm{C}$ (Figure 1). 5 Patients were diagnosed to have Barrett dysplasia by endoscopy 2 of them had GERD and 3 had no GERD.

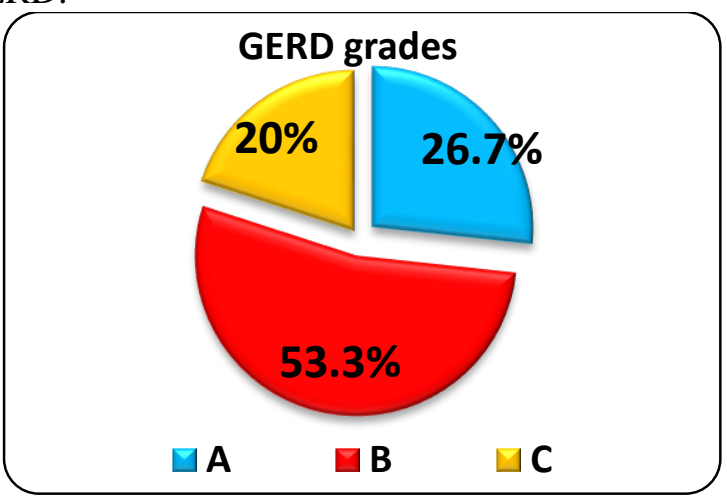

*20\% grade C, $26.7 \%$ grade A, $53.3 \%$ grade B.

Figure 1. GERD grades in studied patients 


\section{Histopathological data:}

As regarding the histopathological data: patients with BE and no dysplasia were 31 (91.2\%) and among 5 patients diagnosed with Barrett's dysplasia by AANBI $3(8.8 \%)$ of them had low grade dysplasia proved on histopathology. The type of barrette epithelium identified on histopathology was $28(82.35 \%)$ had cardiac type BE, $3(8.82 \%)$ had fundic type BE and $3(8.82 \%)$ had intestinal type BE. Among patients with low grade dysplasia all had gastric cardiac type (100\%) of BE. chronic active reflux esophagitis and cardiac $\mathrm{H}$. Pylori infection were recorded in $28(82.4 \%)$ and 8 $(23.5 \%)$ respectively, and among patients with low grade dysplasia all had chronic active reflux esophagitis with no $\mathrm{H}$. pylori recorded in any case. The sensitivity, specificity, positive predictive value, negative predictive value and accuracy of AA-NBI in diagnosis of Barrett's dysplasia were 100\%, 93.5\%, 60\%, 100\%, and $94.1 \%$ respectively (Figure 2 ).

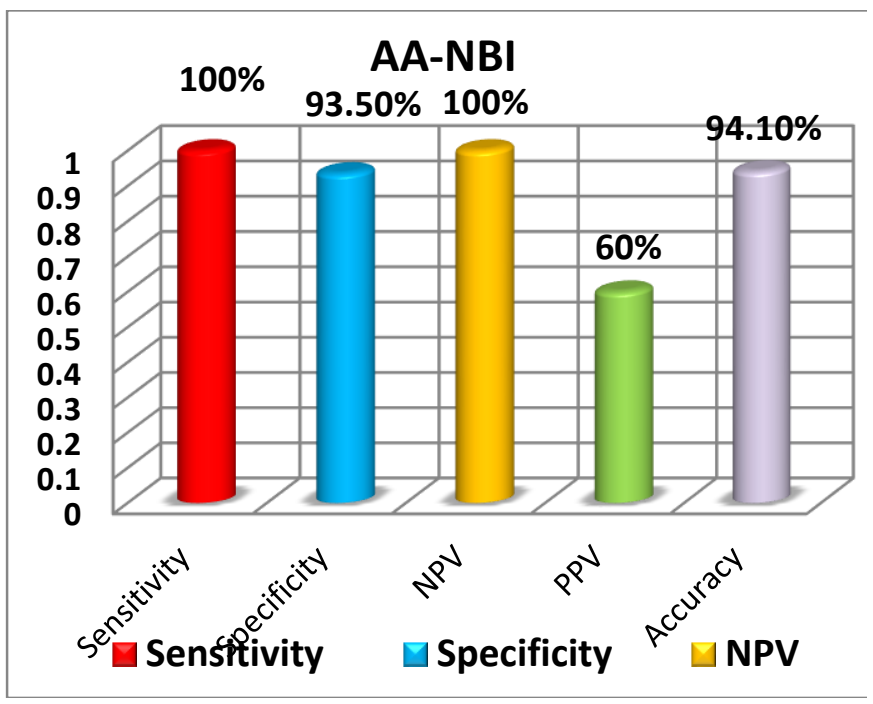

Figure 2. sensitivity, specificity, positive predictive value, negative predicative value and accuracy of NBIAA in prediction of barrett dysplasia.

\section{DISCUSSION}

Our study illustrates that acetic acid chromoendoscopy and narrow band imaging have a promising role in assessment and prediction of esophageal Barrett dysplasia. 34 patients with BE were included in our study; 5 of them were diagnosed by AANBI as Barrett dysplasia based on magnified mucosal pit pattern, magnified vascular pattern and acetowhitening reaction after acetic acid spray over the mucosa. Among the 5 cases diagnosed by endoscopy 3 of them confirmed by histopathology. In contrast, no any cases diagnosed as dysplasia on histopathology and missed by endoscopy.

In our study results demonstrated that the sensitivity and specificity of AA-NBI were $100 \%$ and
93.5\% respectively which are in agreement with a study by Vázquez-Iglesias, et al., which reported high sensitivity $100 \%$ and specificity $97.7 \%$ of detection of dysplasia by acetic acid chromoendoscopy. However, there were only $13 / 100$ of the patient included in the study had dysplasia ${ }^{14}$.

Our findings also consistent with large series of studies by Longcroft-Wheaton, et al., which included 119 patients and concluded that acetic acid-assisted evaluation of Barrett's esophagus detected dysplasia/neoplasia more than white light endoscopy, and showed sensitivity $95.5 \%$ and specificity $80 \%$ which were identical to that of histopathological analysis ${ }^{15}$.

Our study results illustrated that the diagnostic accuracy of AA-NBI for Barrett dysplasia was $94.1 \%$ which is similar to those of Coletta, et al., who reported that acetic acid chromoendoscopy (AAC) has great diagnostic accuracy for recognizing dysplastic Barrett esophagus patients. This study included 13 prospective studies with pooled sensitivity, specificity, positive likelihood ratio, and negative likelihood ratio for all included studies (9 studies, 1379 patients) were 0.92 (95\% confidence interval [CI], 0.83-0.97), 0.96 (95\% CI, 0.85-0.99), 25.0 (95\% CI, 5.9-105.3), and 0.08 (95\% CI, 0.04-0.18), respectively ${ }^{16}$.

In contrast, our findings don't meet the findings of study by Curvers et al., who found that addition of acetic acid chromoendoscopy and narrow band imaging to white light endoscopy didn't increase the yield of recognizing dysplasia/early neoplasia in Barrett esophagus patients ${ }^{17}$.

Sharma et al., reported that narrow band imaging improves detection of dysplasia more than white light image which meets our study results ${ }^{18}$.

Kara et al., also found that narrow band image is superior than standard WLI in detection of Barrett dysplasia in descriptive study including 67 patients using histology as reference standard. In their study, detection of high grade dysplasia had sensitivity $94 \%$, specificity $76 \%$, PPV 64\% and NPV 98\% which are similar to results of our study. But when compared with high-definition white light endoscopy (HDWLE) revealed no differences in the detection rate, however NBI demanded fewer biopsies ${ }^{\mathbf{1 9}}$.

In this study we used NBI BING criteria for identification of dysplasia in patients with Barrett esophagus according to the mucosal pit pattern and vascular pattern and we almost found results similar to results of study done by Sharma et al., which concluded that BING criteria can identify patients with Barrett dysplasia with $85 \%$ accuracy, $80 \%$ sensitivity, $81 \%$ positive predictive value (PPV), and $85 \%$ negative predictive value (NPV). These values were 92\%, 91\%, $89 \%$, and $95 \%$, respectively when dysplasia was evaluated with high confidence ${ }^{\mathbf{1 1}}$. 
In this study there were some limitations. Frist, many patients had esophagitis on endoscopy, which increased rate of false positive endoscopic results for BE. Second, the number of patients with Barrett esophagus included in this study was little (34 patients) and patients with dysplasia was also little (3 patients) making it difficult to draw significant conclusion from it with respect to rate of dysplasia detection.

The high diagnostic accuracy of AA-NBI for detecting and also for excluding Barrett dysplasia suggests that this procedure may be very beneficial to appliance in clinical practice for BE surveillance. AANBI should decrease the number of random biopsies which sometimes can miss dysplasia and facilitate target biopsies increasing yield of dysplasia. Acetic acid chromoendoscopy is safe, low cost, available and don't need specialist training. NBI also is widely available, easily to use with visualization of mucosal and vascular pattern. So, and according to our study results AA-NBI can be used in Barrett esophagus surveillance for Barrett dysplasia.

Our study justifies the need for large study including large number of patients to make a strong conclusion for assessment of the role of AA-NBI in BE surveillance for Barrett's dysplasia.

\section{CONCLUSION}

Nine percent of patients with BE are positive for dysplasia according to our results. Cardiac type columnar metaplasia represents the majority of patients with $\mathrm{BE}$ in our series. AA-NBI has promising sensitivity, specificity and accuracy in prediction of Barrett's dysplasia. Our study justifies the need for large study to assess the role of AA-NBI in BE surveillance for detection of Barrett's dysplasia.

\section{REFERENCES}

1. Wang KK, Sampliner RE (2008): Updated Guidelines 2008 for the Diagnosis, Surveillance and Therapy of Barrett's Esophagus. The American Journal of Gastroenterology, 103 (3): 788-797.

2. Ronkainen J, Aro P, Storskrubb T et al. (2005): Prevalence of Barrett's esophagus in the general population: an endoscopic study. Gastroenterology, 129: 1825-1831.

3. Shaheen NJ, Richter JE (2009): Barrett esophagus. Lancet, 373 (9666): 850-61.

4. De Jonge PJ, van Blankenstein M, Grady WM et al. (2014): Barrett's esophagus: epidemiology, cancer risk and implications for management. Gut, 63: 191-202.

5. Phoa KN, van Vilsteren FG, Weusten BL et al. (2014): Radiofrequency ablation vs endoscopic surveillance for patients with Barrett esophagus and low-grade dysplasia: a randomized clinical trial. Journal of the American Medical Association, 311: 1209-1217.

6. Konda VJ, Ross AS, Ferguson MK et al. (2008): Is the risk of concomitant invasive esophageal cancer in high-grade dysplasia in Barrett's esophagus overestimated? Clin Gastroenterol. Hepatol., 6: 159-164.

7. Sharma P, Gorrepati VS (2018): How Should We Report Endoscopic Results in Patient's with Barrett's Esophagus? Digestive Diseases and Sciences, 63:2115-2121.

8. Fitzgerald RC, di Pietro M, Ragunath K et al. (2014): British Society of Gastroenterology guidelines on the diagnosis and management of Barrett's esophagus. Gut, 63:742.

9. Sturm MB, Wang TD (2015): Emerging optical methods for surveillance of Barrett's esophagus. Gut, 64: 1816-1823.

10. Kandel P, Wallace MP (2017): The Role of Adjunct Imaging in Endoscopic Detection of Dysplasia in Barrett's Esophagus. Gastrointest Endoscopy Clin N Am., 27: 423446.

11. Sharma P, Bergman JJ, Goda K et al. (2016): Development and validation of a classification system to identify high-grade dysplasia and esophageal adenocarcinoma in Barrett's Esophagus using narrow-band imaging. Gastroenterology, 150(3):591-8.

12. Tholoor $S$, Bhattacharyya $R$, Tsagkournis $O$ et al. (2014): Acetic acid chromoendoscopy in Barrett's esophagus surveillance is superior to the standardized random biopsy protocol: results from a large cohort study (with video). Gastrointest Endosc., 80:417-424.

13. Schlemper R J, Riddell R H, Kato Y et al. (2000): The Vienna classification of gastrointestinal epithelial neoplasia. Gut, 47: 251-255.

14. Vázquez-Iglesias JL, Alonso-Aguirre P, Diz-Lois MT et al. (2007): Acetic acid allows effective selection of areas for obtaining biopsy samples in Barrett's esophagus. Eur J Gastroenterol Hepatol., 19:187-193.

15. Longcroft-Wheaton G, Duku M, Mead R et al. (2010): Acetic Acid Spray Is an Effective Tool for the Endoscopic Detection of Neoplasia in Patients with Barrett's Esophagus. clinical gastroenterology and hepatology, 8:843-847.

16. Coletta M, Sami SS, Nachiappan A et al. (2016): Acetic acid chromoendoscopy for the diagnosis of early neoplasia and specialized intestinal metaplasia in Barrett's esophagus: a meta-analysis. Gastrointest Endosc., 83:57-67.

17. Curvers W, Baak L, Kiesslich $R$ et al. (2010): Chromoendoscopy and Narrow-Band Imaging Compared with High-Resolution Magnification Endoscopy in Barrett's Esophagus. Gastroenterology, 134:670-679.

18. Sharma P, Hawes RH, Bansal A et al. (2013): Standard endoscopy with random biopsies versus narrow band imaging targeted biopsies in Barrett's esophagus: a prospective, international, randomized controlled trial. Gut, 62(1):15-21.

19. Kara MA, Ennahachi M, Fockens P et al. (2006): Detection and classification of the mucosal and vascular patterns (mucosal morphology) in Barrett's esophagus by using narrow band imaging. Gastrointest Endosc., 64(2):15566. 